No. 622

December 2019

Simulating two-dimensional viscoelastic fluid flows by means of the "Tensor Diffusion" approach

P. Westervoß, S. Turek

ISSN: 2190-1767 


\title{
Simulating two-dimensional viscoelastic fluid flows by means of the "Tensor Diffusion" approach
}

Patrick Westervoß and Stefan Turek

\begin{abstract}
In this work, the novel "Tensor Diffusion" approach for simulating viscoelastic fluids is proposed, which is based on the idea, that the extra-stress tensor in the momentum equation of the flow model is replaced by a product of the strain-rate tensor and a tensor-valued viscosity. At least for simple flows, this approach offers the possibility to reduce the full nonlinear viscoelastic model to a generalized "Tensor Stokes" problem, avoiding the need of considering a separate stress tensor in the solution process. Besides fully developed channel flows, the "Tensor Diffusion" approach is evaluated as well in the context of general two-dimensional flow configurations, which are simulated by a suitable four-field formulation of the viscoelastic model respecting the "Tensor Diffusion".
\end{abstract}

\section{Introduction}

Numerical simulations of viscoelastic fluids are still a challenging task, especially due to the involved constitutive equations describing the complex material behaviour of the flow. From a numerical point of view, constitutive equations of differential type are quite straightforward to apply in combination with the Stokes equations, but being applicable only for a limited range of flow configurations $[1,2,3]$.

An alternative modelling approach in numerical flow simulations is offered by considering integral constitutive equations, which are often of the so-called timeseparable Rivlin-Sawyers (or Kaye-BKZ) type [4, 5], where the stress tensor is written as an infinite integral of the form

$$
\boldsymbol{\sigma}(t)=\int_{-\infty}^{t} M\left(t-t^{\prime}\right)\left[\phi_{1}\left(I_{1}, I_{2}\right) \mathbf{B}_{t^{\prime}}(t)+\phi_{2}\left(I_{1}, I_{2}\right) \mathbf{B}_{t^{\prime}}(t)^{-1}\right] d t^{\prime}
$$

Institute for Applied Mathematics (LS III), TU Dortmund University, Vogelpothsweg 87, 44227 Dortmund, Germany, e-mail: patrick.westervoss@math.tu-dortmund.de 
In the above stress integral, $\phi_{1}, \phi_{2}$ are empirical functions to model nonlinear effects depending on the two non-trivial invariants $I_{1}, I_{2}$ of the Finger tensor $\mathbf{B}$. One of the most suitable approaches to handle integral material models in combination with the Stokes equations is the so-called "Deformation Fields Method" (DFM, [6, 7, 8]). A central object in this scheme is the Finger tensor, which is evolved in time depending on the velocity field $\mathbf{u}$ according to the differential equation

$$
\frac{\partial}{\partial s} \mathbf{B}_{t^{\prime}}(s)+(\mathbf{u}(s) \cdot \nabla) \mathbf{B}_{t^{\prime}}(s)-\nabla \mathbf{u}(s)^{\top} \cdot \mathbf{B}_{t^{\prime}}(s)-\mathbf{B}_{t^{\prime}}(s) \cdot \nabla \mathbf{u}(s)=\mathbf{0}
$$

in $s \in\left[t^{\prime}, t\right]$ for fixed $t^{\prime}$, where $\mathbf{B}_{t^{\prime}}\left(t^{\prime}\right)=\mathbf{I}$.

However both, the differential as well as integral material model, give rise to numerical challenges due to the complex rheology of the considered viscoelastic fluids. On the one hand, in the differential case, the well-known "High Weissenberg Number Problem" (HWNP, [1, 2]) together with the need of considering multiple modes [3] has to be taken into account. On the other hand, for integral constitutive equations, a suitable numerical treatment of the resulting integro-differential set of equations needs to be derived resp. requires further improvement $[6,7,8]$.

Therefore, in this work, the novel "Tensor Diffusion" approach is introduced, offering the possibility to remove the complex rheology of the fluid from the set of equations and to establish a straightforward numerical treatment of viscoelastic fluids.

\section{The "Tensor Diffusion" approach}

As outlined above, many difficulties and challenges in simulating viscoelastic fluids arise from the complex rheology of the fluid characterized by both, differential and integral constitutive equations. Consequently, avoiding the need of considering such an equation at all would probably improve the general numerical treatment of such fluids. Thus, the underlying assumption of the novel "Tensor Diffusion" approach is the existence of a decomposition of the extra-stress tensor according to

$$
\sigma=\boldsymbol{\mu} \cdot \mathbf{D}(\mathbf{u})
$$

where $\boldsymbol{\mu} \in \mathbb{R}^{2 \times 2}$ in two-dimensional settings. Inserting the stress decomposition (2) into the stationary Stokes equations gives the so-called "Tensor Stokes" problem

$$
-\frac{1}{2} \nabla \cdot\left(\boldsymbol{\mu} \cdot \mathbf{D}(\mathbf{u})+\mathbf{D}(\mathbf{u}) \cdot \boldsymbol{\mu}^{\top}\right)+\nabla p=\mathbf{0}, \quad \nabla \cdot \mathbf{u}=0
$$

Note, that a symmetrized version of the "Tensor Stokes" problem is considered here, since the "Tensor Diffusion" $\boldsymbol{\mu}$ is in general not symmetric as shown in Sect. 3.1 (for details, see $[9,10])$.

Assuming, that the so-called "Tensor Diffusion" $\boldsymbol{\mu}$-corresponding to an actual viscoelastic flow problem - is known or given, the "nonlinear" velocity and pressure solution, originally resulting from the (direct steady) nonlinear differential or 
integral viscoelastic model, can be computed by simply solving the "Tensor Stokes" problem (3) in $(\mathbf{u}, p)$. Thus, the constitutive equation or the complex rheology of such fluids is removed from the system and the corresponding stresses are computed in post-processing fashion based on the velocity solution calculated from Eq. (3). Furthermore, a robust, efficient, accurate and stable numerical scheme can be used for solving the "Tensor Stokes" problem (3), since typical solution techniques for (generalized) Stokes problems, i.e. problems in $(\mathbf{u}, p)$ only, are applicable in this context.

Obviously, the "Tensor Stokes" problem represents an extension of classical generalized Stokes equations involving a shear-rate dependent scalar viscosity (c.f. [11]), since besides the corresponding "shear thinning" effect, in principle the full viscoelastic material behaviour is covered by the tensor-valued viscosity $\boldsymbol{\mu}$ (see Sect. 3). Thus, one of the main potential benefits of the novel "Tensor Diffusion" approach is the possibility to express the complex rheology by a "Tensor Diffusion" $\boldsymbol{\mu}$ instead of solving a nonlinear constitutive equation. In the following section, the validity of this concept will be shown for Poiseuille-like flows, followed by an evaluation for complex flow configurations like the "Flow around cylinder"-benchmark in Sect. 4.

\section{Proof of concept}

In the following, the validity of the underlying assumption, that a stress decomposition according to Eq. (2) exists, is investigated by checking the ability of the "Tensor Diffusion" approach to reproduce viscoelastic flow characteristics usually resulting from differential or integral material models. Therefore, steady-state twodimensional fully developed channel flows for viscoelastic fluids are considered, where the same velocity profile is obtained at any cutline over the channel height, i.e. in $y$-direction. Thus, the velocity field consists only of a $y$-dependent contribution in $x$-direction, i.e. the channel length. Similarly, the components of stress and Finger tensors depend on $y$ only, but not on $x$.

\subsection{Fully developed channel flows for UCM}

Considering the differential steady-state version of the Upper-Convected Maxwell model (UCM, [4]) in the above setting, the corresponding unknowns can be given analytically, especially leading to a parabolic velocity profile. Furthermore, the corresponding (symmetric) strain-rate as well as stress tensors read

$$
\boldsymbol{\sigma}=\left(\begin{array}{ll}
\sigma_{11} & \sigma_{12} \\
\sigma_{12} & \sigma_{22}
\end{array}\right)=\left(\begin{array}{cc}
2 \eta_{p} \Lambda u_{y}^{2} & \eta_{p} u_{y} \\
\eta_{p} u_{y} & 0
\end{array}\right), \mathbf{D}(\mathbf{u})=\frac{1}{2}\left(\begin{array}{cc}
2 u_{x} & v_{x}+u_{y} \\
v_{x}+u_{y} & 2 v_{y}
\end{array}\right)=\left(\begin{array}{cc}
0 & u_{y} \\
u_{y} & 0
\end{array}\right) \text { (4) }
$$

Consequently it is realized, that indeed a matrix- or tensor-valued quantity reading 


$$
\boldsymbol{\mu}=2 \eta_{p}\left(\begin{array}{cc}
1 & 2 \Lambda u_{y} \\
0 & 1
\end{array}\right)
$$

can be derived even analytically, relating $\sigma$ and $\mathbf{D}$ according to Eq. (2).

In principle, the same can be done in case of the steady-state integral version of UCM, where only one single Finger tensor needs to be considered due to the stationary velocity field. However, inserting the analytical expressions for the components of the Finger tensor - derived for fully developed channel flows - into the single-mode "stationary" stress integral for UCM (c.f. [4]) yields

$$
\begin{aligned}
\boldsymbol{\sigma} & =\int_{0}^{\infty} \frac{\eta_{p}}{\Lambda^{2}} \exp \left(-\frac{s}{\Lambda}\right)(\mathbf{B}(s)-\mathbf{I}) d s \\
& =\left[2 \int_{0}^{\infty} \frac{\eta_{p}}{\Lambda^{2}} \exp \left(-\frac{s}{\Lambda}\right)\left(\begin{array}{cc}
s & s^{2} u_{y} \\
0 & s
\end{array}\right) d s\right]\left[\frac{1}{2}\left(\begin{array}{cc}
0 & u_{y} \\
u_{y} & 0
\end{array}\right)\right]=\boldsymbol{\mu} \cdot \mathbf{D}(\mathbf{u})
\end{aligned}
$$

with the same "Tensor Diffusion" $\boldsymbol{\mu}$ as calculated from the differential version. Particularly, a stress decomposition according to Eq. (2) can be derived for differential as well as integral viscoelastic models.

\subsection{Poiseuille-like flow for Wagner model}

In the following, a nonlinear integral model is considered, in detail the Wagner model [12], which - for two-dimensional stationary flow configurations - results in a stress integral of the form

$$
\begin{aligned}
\boldsymbol{\sigma}= & \int_{0}^{\infty} \frac{\eta_{p}}{\Lambda^{2}} \exp \left(-\frac{s}{\Lambda}\right)\left[f \exp \left(-n_{1} \sqrt{I-2}\right)+\ldots\right. \\
& \left.(1-f) \exp \left(-n_{2} \sqrt{I-2}\right)\right] \mathbf{B}(s) d s
\end{aligned}
$$

For fully developed channel flows, the stress integral can be converted into

$$
\sigma=\mu \cdot \mathbf{D}(\mathbf{u})+v
$$

where $\mu, v \in \mathbb{R}^{2 \times 2}$ and

$$
\begin{aligned}
\mu_{11} & =2 \eta_{p}\left[f\left(1+n_{1} \Lambda \sqrt{u_{y}^{2}}\right)^{-2}+(1-f)\left(1+n_{2} \Lambda \sqrt{u_{y}^{2}}\right)^{-2}\right] \\
\mu_{12} & =4 \eta_{p} \Lambda u_{y}\left[f\left(1+n_{1} \Lambda \sqrt{u_{y}^{2}}\right)^{-3}+(1-f)\left(1+n_{2} \Lambda \sqrt{u_{y}^{2}}\right)^{-3}\right] \\
v & =\frac{\eta_{p}}{\Lambda}\left[f\left(1+n_{1} \Lambda \sqrt{u_{y}^{2}}\right)^{-1}+(1-f)\left(1+n_{2} \Lambda \sqrt{u_{y}^{2}}\right)^{-1}\right]
\end{aligned}
$$


besides $\mu_{22}=\mu_{11}$ and $\mu_{21}=0$ as well as $v_{11}=v_{22}=v$ and $v_{12}=v_{21}=0$. Consequently, a "generalized" stress decomposition compared to UCM in Eq. (6) is derived. However, by introducing the modified pressure $P=p-v$, a similar version of the "Tensor Stokes" problem in Eq. (3) is obtained, but now replacing the original pressure $p$ by the modified pressure $P$, since the operator $\nabla \cdot v$ occuring in the "Tensor Stokes" problem can be considered as $\nabla v$ and thus be absorbed into the pressure gradient.

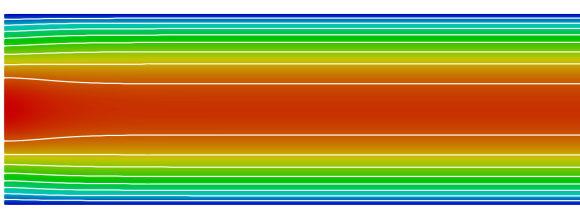

(a) $x$-velocity from $2 \mathrm{D}$

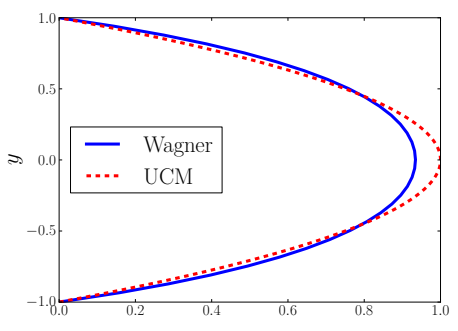

(b) $x$-velocity at $x_{\text {mid }}$

Fig. 1 Channel flow for Wagner model, $\Lambda=1.0, f=0.57, n_{1}=0.31, n_{2}=0.106$

In the following, a modified Poiseuille flow is considerd in Finite Element simulations, where the velocity on in- and outflow edges is set to take a parabolic profile. At the same time, the "Tensor Diffusion" corresponding to a fully developed channel flow is prescribed globally, which is why the flow should evolve to its fully developed nonlinear shape away from in- and outflow.

Obviously, the flow profiles obtained from the Wagner model for the material parameters given in [12] recover the shear-thinning effect regarding the velocity profile as depicted in Fig. 1, which is a typical material behaviour of viscoelastic fluids. Furthermore, this velocity profile, resulting from two-dimensional simulations, matches the solution of the one-dimensional version of the full integral model derived for fully developed channel flows [10]. This indicates, that especially for nonlinear integral models, viscoelastic flow characteristics in fully developed channel flows are reproduced by simply solving a generalized Stokes-like problem of the form (3) in the unknowns $(\mathbf{u}, p)$, where the complex rheology arising from the stress integral is completely hidden in the "Tensor Diffusion".

In principle, the same procedure can be done also for other nonlinear viscoelastic constitutitve equations like the Giesekus model [13] in the differential or the PSM model [14] in the integral case. However, for non of these two cases, the "Tensor Diffusion" $\boldsymbol{\mu}$ can be given in closed form, since it can be derived only "semi analytically" or numerically. But nevertheless, similar results for comparing solutions of one- and two-dimensional simulations can also be obtained for other viscoelastic models than Wagner, further outlining the basic validity of the proposed "Tensor Diffusion" approach [9, 10]. 


\section{Complex flow configurations}

So far, the proposed "Tensor Diffusion" approach is analyzed only in the context of fully developed channel flows, for which it is possible to derive and verify the validity of this novel approach. When more general two-dimensional flow configurations shall be investigated in terms of this novel approach, an explicit derivation of the corresponding tensor-valued viscosity $\boldsymbol{\mu}$ is not (yet?) possible.

Instead, a straightforward implementation for determining the "Tensor Diffusion" numerically is obtained by complementing the original differential steady-state viscoelastic model by an additional algebraic equation regarding $\boldsymbol{\mu}$ and inserting the stress decomposition (2) into the momentum equation of the flow model. Consequently, to evaluate the applicability of the "Tensor Diffusion" approach in the context of general two-dimensional flow configurations, the well-known "Flow around cylinder" benchmark $[1,2,15]$ is simulated by means of the four-field formulation of the above "Tensor Stokes" problem reading

$$
\begin{aligned}
-2 \eta_{s} \mathbf{D}(\mathbf{u})-\frac{1}{2} \nabla \cdot\left(\boldsymbol{\mu} \cdot \mathbf{D}(\mathbf{u})+\mathbf{D}(\mathbf{u}) \cdot \boldsymbol{\mu}^{\top}\right)+\nabla p & =\mathbf{0} \\
\nabla \cdot \mathbf{u} & =0 \\
(\mathbf{u} \cdot \nabla) \boldsymbol{\sigma}-\nabla \mathbf{u}^{\top} \cdot \boldsymbol{\sigma}-\boldsymbol{\sigma} \cdot \nabla \mathbf{u}+\mathbf{f}\left(\Lambda, \eta_{p}, \boldsymbol{\sigma}\right) & =2 \frac{\eta_{p}}{\Lambda} \mathbf{D}(\mathbf{u}) \\
\boldsymbol{\mu} \cdot \mathbf{D}(\mathbf{u})-\boldsymbol{\sigma} & =0
\end{aligned}
$$

which is discretized within the Finite Element framework presented in [2], where the "Tensor Diffusion" is approximated by elementwise constant polynomials [10].

Within the typical benchmark configuration of a present solvent contribution of $\eta_{s}=0.59$, the drag coefficients $C_{D}(\mathbf{T})$, which are computed based on the total stress tensor $\mathbf{T}$, are analyzed for evaluating the quality of the simulation results for several Weissenberg numbers $\mathrm{We}=\Lambda U_{\text {mean }} / R$. Therefore, the drag coefficients calculated from the "Tensor Diffusion" are compared to reference results as well as results based on the original approach validated in [2]. In the following, $\mathbf{T}_{\sigma}$ denotes the total stress tensor arising from the original viscoelastic model and $\mathbf{T}_{\mu}$ the one corresponding to the "Tensor Stokes" problem, where in principle $\sigma$ is replaced by the symmetrized stress-decomposition to obtain $\mathbf{T}_{\mu}$ from $\mathbf{T}_{\sigma}$.

Table 1 Oldroyd-B model $[4,16]$

\begin{tabular}{cccc}
\hline We $C_{D}\left(\mathbf{T}_{\sigma}\right)$ & $C_{\boldsymbol{D}}\left(\mathbf{T}_{\boldsymbol{\mu}}\right)$ & Ref. [1] \\
\hline 0.1 & 130.342 & 130.348 & 130.36 \\
0.2 & 126.605 & 126.624 & 126.62 \\
0.3 & 123.172 & 123.212 & 123.19 \\
0.4 & 120.553 & 120.549 & 120.59 \\
0.5 & 118.747 & 118.751 & 118.83
\end{tabular}

Table 2 Giesekus model, $\alpha=0.1$ [13]

\begin{tabular}{cccc}
\hline We & $C_{D}\left(\mathbf{T}_{\sigma}\right)$ & $C_{D}\left(\mathbf{T}_{\mu}\right)$ & Ref. [15] \\
\hline 0.1 & 125.567 & 125.572 & 125.58 \\
0.5 & 103.717 & 103.733 & 103.73 \\
1.0 & 95.536 & 95.568 & 95.55 \\
5.0 & 85.210 & 85.243 & - \\
10.0 & 83.047 & 83.068 & - \\
\hline
\end{tabular}


A summary of the drag coefficients resulting from the above configuration is given in Tab. 1 and 2, which illustrates, that the drag coefficients obtained from the four-field formulation (10) of the "Tensor Stokes" problem show a good agreement to the results computed by means of the original method as well as the reference results $[1,15]$ for both, Oldroyd-B and Giesekus model. For the latter, reference results apparently are available only up to We $=1.0$, which is why the "Tensor Stokes" results for higher Weissenberg numbers are evaluated by a comparison with the original approach only.

The more challenging configuration is represented by considering the "no solvent" case in the above setting, where $\eta_{s}=0$ in Eq. (10a). Unfortunately, no reference results are available for this flow configuration, which is why the "Tensor Stokes" results are again compared only against the results of the original approach.

Table 3 UCM $(\alpha=0.0)$ or Giesekus model

\begin{tabular}{cccc}
\hline We & $\alpha$ & $C_{D}\left(\mathbf{T}_{\sigma}\right)$ & $C_{D}\left(\mathbf{T}_{\mu}\right)$ \\
\hline 0.1 & 0.0 & 127.373 & 127.403 \\
0.5 & 0.0 & 96.046 & 98.054 \\
\hline 0.1 & 0.1 & 115.377 & 115.508 \\
0.5 & 0.1 & 60.804 & 61.992 \\
\hline
\end{tabular}

When analyzing the calculated drag coefficients given in Tab. 3, again the "Tensor Stokes" results show a good agreement to the results of the original problem especially for lower We for both, the UCM as well as Giesekus model. Besides, for the Giesekus model it was not possible to reach significantly larger Weissenberg numbers as in the case of UCM, which again illustrates the complexity of this flow configuration.

Additionally, recall that $\boldsymbol{\mu}$ is approximated in $Q_{0}$ only, which is of lower order than the corresponding approximation of $\sigma$ in $Q_{2}$. Naturally, results obtained from the original problem are expected to be of higher accuracy anyway. But nevertheless, applying the "Tensor Diffusion" approach gives simulation results of a similar quality as the original approach, even for this complex flow configuration.

\section{Conclusion}

In this work, the novel "Tensor Diffusion" approach is introduced, where in principle the extra-stress tensor in the momentum equation of the viscoelastic model is replaced by a product of the so-called "Tensor Diffusion" and the strain-rate tensor.

The underlying assumption, that such a stress decomposition exists in general, is verified in a first step for fully developed channel flows, where the full viscoelastic model can be reduced to a so-called "Tensor Stokes" problem. Consequently, the nonlinear viscoelastic solution might be simply computed from a generalized Stokeslike problem including a tensor-valued viscosity. 
Furthermore, the applicability of the "Tensor Diffusion" approach is evaluated within the two-dimensional "Flow around cylinder" benchmark. Here, the drag coefficients resulting from the original viscoelastic model as well as reference results are reproduced quite well. But nevertheless, as a main goal of future work, the full viscoelastic flow model shall be reduced to a pure "Tensor Stokes" problem.

\section{References}

1. M. A. Hulsen, R. Fattal, R. Kupferman, Flow of viscoelastic fluids past a cylinder at high weissenberg number: Stabilized simulations using matrix logarithms, Journal of Non-Newtonian Fluid Mechanics 127 (1) (2005) 27 - 39. doi:https://doi.org/10.1016/j.jnnfm.2005.01.002.

2. H. Damanik, J. Hron, A. Ouazzi, S. Turek, A monolithic fem approach for the log-conformation reformulation (lcr) of viscoelastic flow problems, Journal of Non-Newtonian Fluid Mechanics 165 (19) (2010) 1105 - 1113. doi:https://doi.org/10.1016/j.jnnfm.2010.05.008.

3. J. Kroll, S. Turek, P. Westervoß, Evaluation of nonlinear differential models for the simulation of polymer melts, Kautschuk Gummi Kunststoffe (317) (2017) 48-52.

4. R. G. Larson, Constitutive Equations for Polymer Melts and Solutions, Butterworths Series in Chemical Engineering, Butterworth-Heinemann, 1988.

5. R. Keunings, Finite element methods for integral viscoelastic fluids, 2003.

6. E. Peters, M. Hulsen, B. van den Brule, Instationary eulerian viscoelastic flow simulations using time separable rivlin-sawyers constitutive equations, Journal of Non-Newtonian Fluid Mechanics 89 (1) (2000) 209 - 228. doi:https://doi.org/10.1016/S0377-0257(99)00026-9.

7. M. Hulsen, E. Peters, B. van den Brule, A new approach to the deformation fields method for solving complex flows using integral constitutive equations, Journal of Non-Newtonian Fluid Mechanics 98 (2) (2001) 201 - 221. doi:https://doi.org/10.1016/S0377-0257(01)00110-0.

8. M. A. Hulsen, P. D. Anderson, The deformation fields method revisited: Stable simulation of instationary viscoelastic fluid flow using integral models, Journal of Non-Newtonian Fluid Mechanics 262 (2018) 68 - 78. doi:https://doi.org/10.1016/j.jnnfm.2018.03.001.

9. P. Westervoß, S. Turek, H. Damanik, A. Ouazzi, The "tensor diffusion" approach for simulating viscoelastic fluids, Tech. rep., Department of Mathematics, technical report of the Institute for Applied Mathematics, No. 617 (Nov. 2019). doi:http://dx.doi.org/10.17877/DE290R-20363.

10. P. Westervoß, A new approach for simulating viscoelastic fluids, Ph.D. thesis, TU Dortmund (to be submitted in 2020).

11. A. Ouazzi, S. Turek, Numerical methods and simulation techniques for flow with shear and pressure dependent viscosity, in: M. Feistauer, V. Dolejsi, P. Knobloch, K. Najzar (Eds.), Numerical Mathematics and Advanced Applications, Springer, 2003, pp. 668-676, enumath 2003 Prague; ISBN-Nr. 3-540-21460-7.

12. H. M. Laun, Description of the non-linear shear behaviour of a low density polyethylene melt by means of an experimentally determined strain dependent memory function, Rheologica Acta 17 (1) (1978) 1-15. doi:10.1007/BF01567859.

13. H. Giesekus, Die elastizität von flüssigkeiten, Rheologica Acta 5 (1966) 29-35.

14. C. W. Macosko, R. G. Larson, K. (Firm), Rheology : principles, measurements, and applications, New York : VCH, 1994, originally published as ISBN 1560815795.

15. S. Claus, T. Phillips, Viscoelastic flow around a confined cylinder using spectral/hp element methods, Journal of Non-Newtonian Fluid Mechanics 200 (2013) 131 - 146, special Issue: Advances in Numerical Methods for Non-Newtonian Flows. doi:https://doi.org/10.1016/j.jnnfm.2013.03.004.

16. J. G. Oldroyd, On the formulation of rheological equations of state, Proceedings of the Royal Society of London. Series A, Mathematical and Physical Sciences 200 (1063) (1950) 523-541. 